\title{
Artificial Intelligence in the Fight Against COVID-19 in the Banking Sector
}

\section{Hazem Shatila}

Bradley Department of electrical and computer engineering, Virginia Polytechnic Institute and State University, USA

\author{
${ }^{*}$ Corresponding author \\ Hazem Shatila, Bradley Department of electrical and computer engineering, \\ Virginia Polytechnic Institute and State University, USA
}

Submitted: 16 Sept 2020; Accepted: 26 Sept 2020; Published: 15 Oct 2020
The COVID-19 pandemic has been causing a massive strain in different sectors around the globe, especially in the health care systems in many countries. Artificial Intelligence has found its way in the health care system in helping to find a cure or vaccine by screening out medicines that could be promising for cure. Not only that but by containing the virus and predicting highly effected areas and limiting the spread of the virus. Many use cases based on AI was successful to monitor the spread and lock areas that were predicted by AI algorithms to be at high risk. Broadly speaking, AI involves 'the ability of machines to emulate human thinking, reasoning and decision - making'

As the whole world, at the moment, are making use of all available digital services from buying on-line, on-line teaching at schools and universities...etc., the financial sector was flagged with the importance of increasing their digital services for their customers. Banks that had powerful and mature digital services has a much easier burden to support their customers, banks lacking behind in digital maturity are having a much harder time. The importance of digital transformation in the banking sector is currently clear now, more than ever before. Artificial Intelligence is a key player in the roadmap of digital transformation, and its application in the banking sector has been there for a while. AI is now at test during this Pandemic!!! The World Economic Forum has said that " $A I$ is going to be very valuable in the fight against the coronavirus".

The good news is that banks have been working to digitize their services for decades now. The work that was done in the past to digitize most of the experiences and processes is serving the banking sector well now. Banks at this crisis are seeing a surge in activity digitally as clients are choosing to interact with them in this way. In developing countries, people need more credit and data advances to stay in contact during these challenging times, as well as contactless micro-finance services to replace physical transactions. Managing this load using the already stretched infrastructure of such markets is a significant challenge, but one where machine learning, big-data analytics and artificial intelligence can really make a difference. Mobile solutions are proving to be a lifeline in emerging economies. Fintech firms can use this opportunity to build their reputations and emerge stronger once the crisis has passed.
According to a report by Bloomberg, listings for AI-based jobs within the financial sector increased by approximately $60 \%$ from 2018 to 2019 and expected to exceed more in 2020. This demand for workers with AI expertise is not only seen within the financial industry but across a variety of other professional sectors, such as e-commerce, digital marketing and social media. This 'skills shortage' is a major obstacle to the adoption of AI in business, with $51 \%$ of those surveyed acknowledging that they don't have enough individuals trained in-house to make their strategies a reality. Machine learning can offer benefits in many forms and different businesses have varying needs. There is no 'one size fits all approach' when adopting and deploying AI, which can make it a costly process for many organizations not equipped with the right tools.

Fortunately, there is ample opportunity to enhance the responsibilities of numerous roles within their organization or let employees get on with more strategic work. SEB, a large Swedish bank, uses a virtual assistant called Aida which is able to handle natural-language conversations and so can answer a trove of customer FAQs. This means customer service professional has been redeployed to focus on complex requests and their more meaningful responsibilities while reducing more customer contact. Bank of America, Capital One, and others have also AI enabled chatbots to help their customers with a variety of tasks. Chatbots are able to engage and interact with customers 24/7 breaking down the idea of traditional banking hours and making less physical interaction with customers and banking personal. This also has led them into figuring out how they can customize and personalize the online experience for each customer. Use cases include personalized content, personalized advice or guidance, behavior profiling, recommendation systems, and other ways of bringing information and data together specifically for the benefit of an individual.

One of the biggest use cases of artificial intelligence within financial services is fraud protection. With the rise of online banking and the exponential growth of digital payments which even increased more due to the pandemic, banks have to monitor huge swathes of transactions for fraudulent behavior. This huge 
influx of data points poses major issues for the human brain but actually maximizes the effectiveness of machine learning systems. We've seen significant growth in the use of deep learning, with most major retail banks now relying on machine learning tools to recognize and flag suspicious activity. To keep up with the pace of criminals and comply with stricter regulations, service providers have to look beyond traditional methods and implement hybrid strategies built around holistic understandings of behavioral and anomalous data.

The COVID-19 pandemic has severely impacted small businesses across the world, National and State governments are offering financial assistance in the form of relief loan packages, designed to help small businesses navigate the crisis. Small business owners are applying for loans through their banks to keep their workers on the payroll during the pandemic. This has led to banks getting inundated with a massive volume of loan application requests from small businesses, all of which must be reviewed and approved in a short time, which is practically impossible for loan officers to handle. Using technologies in Artificial Intelligence like computer vision, NLP \& machine learning to extract critical data from multiple documents such as loan application forms, was done very quickly so that quicker and faster decisions can be taken by loan officers.

Artificial intelligence and machine learning have already enhanced numerous capabilities for the financial sector, improving recommendations, customer experience, and efficiencies via automation. AI will continue to dominate different parts of the financial sector, and the acquisition of machine learning and data science talent will become the norm. A recent survey from the World Economic Forum attests to this, with nearly two-thirds of financial services leaders expecting to be mass adopters of AI in two years compared to just $16 \%$ today.
Acquiring the right talent to drive machine learning and $\mathrm{AI}$ in organizations will remain a challenge as innovation is focused in different areas and new technologies are being implemented. In lockstep with this will be the constantly evolving regulatory landscape surrounding adoption of AI in financial services as each side races to match and often contain the other. However, the multiple benefits that come from implementing AI and machine learning are clear, and it will be a key area of focus and growth for businesses within financial services over the next decade.

The pandemic-induced developments in digital banking services are not fleeting. While the impacts of the coronavirus will lessen over time, the lessons learned for consumers and institutions alike will only deepen the reliance on digital services across all industries. Most banks and credit unions were ready for this type of need. However, these ever-changing innovations continue to require the best-in-class technology and support. While nobody can be certain about what the post-coronavirus world will look like, there is one reality that has been proven without a doubt, that digital transformation, data and advanced analytics using artificial intelligence and machine learning will be a top priority in any strategic roadmap for any financial institute.

Copyright: @2020 Hazem Shatila. This is an open-access article distributed under the terms of the Creative Commons Attribution License, which permits unrestricted use, distribution, and reproduction in any medium, provided the original author and source are credited. 